\title{
Increase in articles published by authors from Mainland Chinese hospitals in high-impact journals: a comparison between 2012 and 2017
}

\author{
Mina Min Wü, Joy Qingjiao Liao , John Shibing Zhang, Elva Kangyan Chen, Selin Hua He, \\ Yuan-Yuan Zhang* and Harry Hua-Xiang Xia*
}

The research outputs have rarely been studied in Mainland China. The present study aimed to analyse research articles published in high-impact journals (HIJs) by authors from Mainland Chinese hospitals in 2012 and 2017. All types of articles were searched in the PubMed and Embase databases. The durations were 1 January-31 December 2012 and 1 January-31 December 2017. Ten parameters were analysed. Overall, 205 and 871 articles were published in 2012 and 2017 respectively, in 198 HIJs. The proportion of articles published in journals with journal impact factor $(J I F)>25$ was higher in 2017 than in 2012 (12.3\% vs 9.8\%). The number (proportion) of original articles increased from $146(71.2 \%)$ in 2012 to 509 (58.4\%) in 2017. There were 92 (44.9\%) and 89 (43.4\%) articles in basic and clinical research in 2012, whereas the numbers (proportions) were $409(44.9 \%)$ and $230(26.4 \%)$ respectively, in 2017. The number of articles acknowledging domestic (within China) and international (between Mainland China and other countries) cooperation in 2017 increased by almost seven-fold (281 vs 41) and eight-fold (289 vs 38) respectively. Funding acknowledgement was made in 85.4\% and 75.8\% of articles in 2012 and 2017 respectively. The number of articles published in HIJs by authors from Mainland Chinese hospitals has increased rapidly from 2012 to 2017, with only approximately 10\% being published in journals with JIF > 25. The number of original articles on basic and clinical research has dramatically increased. Domestic and international cooperation and research funding are found to play an important role.

Keywords: Biomedical research, high-impact journals, scientific articles.

SCIENTIFIC research articles are an important aspect of scientific research output that show the inherent quality and level of research. The number and quality of articles are the two important indicators. Currently, the quality of articles is reflected by the widespread accepted journal

\footnotetext{
Mina Min Wu, Joy Qingjiao Liao, John Shibing Zhang, Elva Kangyan Chen, Selin Hua He and Harry Hua-Xiang Xia are in Medjaden Bioscience Limited, Room 1201, Block D, Jinyuan World Center, 21 Yue Fei Street, Zhongshan Road, Jiang'an District, Wuhan, China; YuanYuan Zhang is in the Department of Pharmacology, West China Schoo of Preclinical and Forensic Medicine, Sichuan University, 17 Section 3, Renmin Nanlu, Chengdu, China; Harry Hua-Xiang Xia is also in the Department of Gastroenterology, the First Affiliated Hospital of Guangdong Pharmaceutical University, 19 Longlinxia Road, Yuexiu District, Guangzhou, China.

*For correspondence. (e-mail: sarahyyzhang@hotmail.com; harryxia@medjaden.com)

${ }^{\#}$ Contributed equally.
}

impact factor (JIF), an index included in the Journal Citation Reports (JCR) published by Clarivate Analytics, which takes into account the outbound cited references from any of the five journal and proceedings indexes in the Web of Science ${ }^{1}$. Therefore, JIF has become the most important indicator of research quality ${ }^{2}$.

In Mainland China, the investment on scientific research has been growing by double digits each year since the implementation of the Reform and Opening-up policy in 1978. In 2016, investments reached 1.54 trillion RMB, accounting for $2.1 \%$ of the gross domestic product ${ }^{3}$. In 2016, China ranked second in the total number of publications and total citations, but only 15 th in the average number of citations per article 4 . These data indicate that the quality of articles published by Chinese authors needs improvement.

Only a few studies have analysed scientific publications from China. Cheng ${ }^{5}$ and Wang et al. ${ }^{6}$ reported that 
the overall number of articles in orthopedics had significantly increased between 2000 and 2012; however. Liao et $a l .^{7}$ analysed the perceptions of Chinese biomedical researchers towards academic misconduct and reported that participants estimated that approximately $40 \%$ of published scientific articles were associated with some form of academic misconduct. However, there is no study specifically focusing on the scientific articles published in high-impact journals (HIJs) by Chinese authors.

Biomedical research has attracted a large amount of investment in China. In 2017, 38.6\% of the total fund from the National Natural Science Foundation of China (NSFC) was allocated to biomedical research ${ }^{8}$. It is conceivable that the quality of scientific articles in biomedical research may also represent the level of scientific and technical advances in general in China, where researchers in the hospitals have contributed to most scientific articles in biomedical research ${ }^{9}$. Thus, analysis of scientific research articles published in HIJs by researchers working in Chinese hospitals would reflect the status and trend of biomedical research in China.

Therefore, the aim of this study was to analyse the scientific research articles published in HIJs by researchers who were listed as the first or corresponding authors and working in Mainland Chinese hospitals, with strategic recommendations to the researchers, as well as their affiliated hospitals, on how to publish articles in HIJs.

\section{Methods}

\section{Definition of a high-impact journals and inclusion criteria}

In the present study, a journal included in JCR 2017 and having a JIF of 10 or greater is defined as a HIJ.

The inclusion criteria for articles analysed in the present study were: (i) All types of articles, including original articles, review articles, case reports, letters, images, editorials, correspondence items, perspectives, etc. that were published in HIJs and (ii) A Mainland Chinese hospital as the affiliation of the first or corresponding author(s).

\section{Search strategies}

First, 198 journals with JIF of 10 or above in 2017 were identified from the $J C R$, according to the above-mentioned inclusion criteria. Then, a computerized literature search for articles that met the inclusion criteria described above was conducted in the PubMed and Embase databases. The durations were 1 January-31 December 2012 and 1 January-31 December 2017. The search terms were China [affiliation], AND Hospital [affiliation], AND Year [Date], AND ISSN of the Journals [Journal]. The detailed search terms are listed in the Supplementary material.

\section{Data extraction}

Search results were independently screened for eligibility by two reviewers (M. Wu and J. Liao) using the author affiliations. Discrepancies were resolved by consensus through discussions between the two reviewers. If a consensus was not reached, a third reviewer made the final decision. Once a publication was found to meet the inclusion criteria, all required information was collected, including name, ISSN and JIF, title and abstract of the article, publication year, the first/corresponding authors and their affiliations, research field/specialty, type of research, type of article, and source of funding acknowledged.

\section{Variables analysed}

A bibliometric analysis was then performed on the following parameters:

(1) Number of articles in 2012 and 2017: The total number of articles included in 2012 and 2017.

(2) Distribution of JIF for the articles: The number of articles published in journals with JIF of 10-15, 15-25, 25-40, 40-55, and more than 55.

(3) Type of article: The number of different types of articles including original, review, meta, letter and others (e.g., case report, image, correspondence, perspective, etc.).

(4) Type of research: The number of articles in basic and clinical research.

(5) The number of articles in various research fields: The number of articles in various research fields where authors from Chinese hospitals published mostly highimpact articles in 2012 and 2017. Data on the top 10 research fields were ranked in 2017 where Chinese authors published mostly high-impact articles.

(6) The number of articles with domestic and international cooperation: According to the affiliations of the corresponding authors or first authors, the number of articles in relation to domestic and international cooperation. Domestic cooperation was considered if the authors were from two or more different affiliations within China, including those in Mainland China, Hong Kong, Taiwan and Macao, and international cooperation was considered if any author(s) came from an affiliation of a country outside of China. An article with domestic and international cooperation was counted under international cooperation. Otherwise, no cooperation was considered.

(7) Source of funding: According to information in the acknowledgement section, the source of funding was classified into four categories, including: (i) Key Basic Research Foundation, (ii) NSFC, (iii) provincial and municipal funding, and (iv) others. The Key Basic Research Foundation included the National Basic Research 
Programme of China, the National High-tech R\&D Programme of China, and other national projects. Others included the Ministry of Education funding, institutional funding and international funding.

(8) The top 10 journals that published mostly Chinese articles: The number of articles published in HIJs. According to these numbers, journals were ranked to select the top 10 journals that published mostly Chinese articles.

(9) The number of articles published in the top 10 journals with highest JIF. The top 10 journals in 2017 were CA: A Cancer Journal for Clinicians (impact factor (IF): 187.040), New England Journal of Medicine (IF: 72.406), Nature Reviews Drug Discovery (IF: 57.000), Chemical Reviews (IF: 47.928), Lancet (IF: 47.831), Nature Reviews Molecular Cell Biology (IF: 46.602), Journal of the American Medical Association (IF: 44.405), Nature Biotechnology (IF: 41.667), Nature Reviews Genetics (IF: 40.282) and Nature (IF: 40.137).

(10) The number of articles published in the top four popular general medical journals. These are New England Journals of Medicine (NEJM), Journal of the American Medical Association (JAMA), Lancet and British Medical Journal (BMJ). We also considered the number of articles published in Cell, Nature and Science.

\section{Results}

\section{Number of articles in 2012 and 2017 and the distribution of journal impact factors}

The total number of articles published in these 198 journals by authors as first or corresponding authors from Chinese hospitals was 205 in 2012 and 871 in 2017 (Supplementary material). Full texts of all articles were obtained.

As shown in Table 1, 59.5\% $(n=122)$ and $53.8 \%$ $(n=469)$ of the articles were published in journals with JIF $10-15$ in 2012 and 2017 respectively, and $9.8 \%$ $(n=20)$ and $12.3 \%(n=107)$ of the articles were published in journals with JIF > 25 in 2012 and 2017 respectively.

\section{Type of article and type of research}

As shown in Table 1, the number of original and review articles increased from 146 and 17 in 2012 to 509 and 44 in 2017 respectively, but the proportion decreased from $71.2 \%$ and $8.3 \%$ in 2012 to $58.4 \%$ and $5.1 \%$ in 2017 respectively. The number of meta-analysis articles was 15 in both 2012 and 2017, with the proportion being $7.3 \%$ and $1.7 \%$ in 2012 and 2017 respectively. It is noticeable that the number (proportion) of letters increased dramatically from $1(0.5 \%)$ in 2012 to $137(15.7 \%)$ in 2017 . The number and proportion of other types of articles, such as case reports, images, editorials, correspondence items, perspectives, etc. also increased from 2012 to 2017 (26 vs 166 and $12.7 \%$ vs $19.1 \%$ respectively).

As shown in Table 1, there were 92 (44.9\%) articles in basic research and $89(43.4 \%)$ in clinical research in 2012; the number (proportion) increased to 409 (47.0\%) and $230(26.4 \%)$ respectively, in 2017. In other words, whereas the number of the articles in basic and clinical research was similar in 2012, it was about 1.7 times more in basic research than that in clinical research in 2017.

\section{Articles in various research fields}

Table 2 shows the top 10 research fields, as ranked in 2017, where Chinese authors mostly published highimpact articles. The top three research fields were gastroenterology and hepatology, cardiac and cardiovascular systems, and oncology in 2012, and gastroenterology and hepatology, medicine (general and internal), and multidisciplinary sciences in 2017.

Since Chinese authors published most high-impact articles in gastroenterology and hepatology in both 2012 and 2017, we further analysed the articles that were published in the top four journals in gastroenterology and hepatology. In 2012, 14, 3, 13 and 12 articles were published by Chinese authors in Gastroenterology, Gut, Hepatology and Journal of Hepatology, accounting for $2.6 \%, 0.9 \%, 2.4 \%$ and $2.4 \%$ of the total number of the articles published by these journals respectively. The number (proportion) increased to $37(6.4 \%), 27(8.9 \%)$, $67(13.1 \%)$ and $23(6.1 \%)$ respectively, in 2017.

Table 1. Distribution of articles published in high-impact journals (HIJs) by authors from Mainland Chinese hospitals in terms of impact factor, article type and research type

\begin{tabular}{lcc}
\hline & $\begin{array}{c}\text { Number (proportion) } \\
\text { of articles in 2012 } \\
(n=205)\end{array}$ & $\begin{array}{c}\text { Number (proportion) } \\
\text { of articles in 2017 } \\
(n=871)\end{array}$ \\
\hline Impact factor & $122(59.5 \%)$ & $469(53.8 \%)$ \\
$10-15$ & $63(30.7 \%)$ & $295(33.9 \%)$ \\
$15-25$ & $11(5.4 \%)$ & $45(5.2 \%)$ \\
$25-40$ & $8(3.95 \%)$ & $37(4.2 \%)$ \\
$40-55$ & $1(0.5 \%)$ & $25(2.9 \%)$ \\
$>55$ & $146(71.2 \%)$ & $509(58.4 \%)$ \\
Article type & $17(8.3 \%)$ & $44(5.1 \%)$ \\
Original & $15(7.3 \%)$ & $15(1.7 \%)$ \\
Review & $1(0.5 \%)$ & $137(15.7 \%)$ \\
Meta & $26(12.7 \%)$ & $166(19.1 \%)$ \\
Letter & & $409(47.0 \%)$ \\
Others* & $92(44.9 \%)$ & $230(26.4 \%)$ \\
Research type & $89(43.4 \%)$ & $232(26.6 \%)$ \\
Basic research & $24(11.7 \%)$ & \\
Clinical research & & \\
Others** & &
\end{tabular}

*Includes case reports, images, correspondence items, perspectives, etc. **Refers to articles that cannot be classified as either basic or clinical research, such as review, letter, etc. 
Table 2. Top 10 research fields in which authors from Mainland Chinese hospitals published articles in HIJs in 2012 and 2017

\begin{tabular}{|c|c|c|c|c|}
\hline \multirow[b]{2}{*}{ Rank } & \multicolumn{2}{|l|}{2012} & \multicolumn{2}{|c|}{2017} \\
\hline & Research field & Number of Chinese articles & Research field & Number of Chinese articles \\
\hline 1 & Gastroenterology and hepatology & 43 & Gastroenterology and hepatology & 155 \\
\hline 2 & Cardiac and cardiovascular systems & 25 & Medicine, general and internal & 79 \\
\hline 3 & Oncology & 22 & Multidisciplinary sciences & 79 \\
\hline 4 & Hematology & 18 & Cardiac and cardiovascular systems & 72 \\
\hline 5 & Endocrinology and metabolism & 15 & Materials science, multidisciplinary & 51 \\
\hline 6 & Respiratory system & 11 & Oncology & 49 \\
\hline 7 & Cell biology & 10 & Biochemistry and molecular biology & 49 \\
\hline 8 & Medicine, general and internal & 7 & Endocrinology and metabolism & 39 \\
\hline 9 & Biochemistry and molecular biology & 7 & Cell biology & 36 \\
\hline 10 & Urology and nephrology & 6 & Hematology & 35 \\
\hline
\end{tabular}

\section{Domestic and international cooperation and sources of funding}

The number (proportion) of articles with domestic cooperation increased from $41(20.0 \%)$ in 2012 to 281 $(32.3 \%)$ in 2017 . The number (proportion) of articles with international cooperation also increased from 38 (18.5\%) in 2012 to 289 (33.2\%) in 2017.

Table 3 shows the number and proportion of the articles that acknowledged grant funding. Funding acknowledgement was made in $85.4 \%(n=175)$ and $75.8 \%$ $(n=660)$ of the articles respectively; the rates were $96.6 \%(n=141)$ and $96.8 \%(n=499)$ for original articles and $94.6 \%(n=87)$ and $99.8 \%(n=408)$ for articles in basic research respectively. NSFC funding was acknowledged in $60.0 \%(n=123)$ and $55.0 \%(n=479)$ of the articles in 2012 and 2017 respectively. In addition, funding from other sources, including Ministry of Education funding, institutional funding and international funding was acknowledged in $54.1 \% \quad(n=111)$ and $52.6 \%$ $(n=458)$ of the articles in 2012 and 2017 respectively.

\section{Top 10 journals that published mostly Chinese} articles and number of Chinese articles published in the top 10 journals in terms of journal impact factor

Table 4 shows the list of 10 high JIF journals that published mostly Chinese articles in the two years; 10 and 9 of these journals had JIF lower than 20 in 2012 and 2017 respectively. Among these journals, three were in gastroenterology and hepatology in 2012, whereas there were four in 2017.

Table 5 shows the number of Chinese articles in the top 10 journals. Notably, the number (proportion) of articles published in NEJM increased from zero $(0.0 \%)$ in 2012 to $24(1.6 \%)$ in 2017. Also, the number (proportion) of articles published in Lancet and JAMA increased from four $(0.2 \%)$ and three $(0.3 \%)$ respectively, in 2012 to 15 $(1.0 \%)$ and $17(1.5 \%)$ in 2017 respectively. In addition, the number (proportion) of articles published in Nature increased from one $(0.05 \%)$ in 2012 to five $(0.2 \%)$ in 2017.

\section{Articles published in the four popular general medical journals, and Cell, Nature and Science}

Table 5 shows the number of the articles published in NEJM, Lancet, JAMA and BMJ. The number of articles published in $N E J M$ was 0 in 2012 and 24 in 2017. Of the 24 articles, 12 were clinical studies (seven originals, five images), and the rest were letters, correspondence items, etc. The seven originals were prospective clinical trials with large samples. The number of articles published in Lancet was four (two clinical studies, including one original and one case report and two others) in 2012 and 15 (4 clinical studies, including 2 originals and 2 images and 11 others) in 2017. All the three originals published in 2012 and 2017 were national surveys with significant medical importance. The number of articles published in JAMA was three (all clinical studies, including one original and two images) in 2012 and 17 (4 originals in clinical research and 13 others) in 2017. All the five originals were multicentre RCTs. The number of articles published in $B M J$ was zero in 2012 and 12 in 2017 . However, none of these articles was original. Taken together, among all 75 articles published in these four journals in the two years, only $15(20.0 \%)$ were original articles, and the rest $(80.0 \%)$ were meta-analyses, letters, case reports, images, etc.

In 2012, there was one article published in Nature, and no article was published in Science and Cell. In 2017, five, one, and six articles were published in Nature, Science and Cell respectively.

\section{Discussion}

The present study shows that the number of high-impact articles published by researchers from the Mainland Chinese hospitals has increased rapidly from 2012 to 2017. 
GENERAL ARTICLES

Table 3. Number and proportion of articles that acknowledged research grant funding

\begin{tabular}{lcr}
\hline Funding acknowledgement & Number (proportion) of articles in 2012 $(n=205)$ & Number (proportion) of articles in 2017 $(n=871)$ \\
\hline Yes* & $175(85.4 \%)$ & $660(75.4 \%)$ \\
Key Basic Research Foundation** & $62(30.2 \%)$ & $208(23.9 \%)$ \\
NSFC & $123(60.0 \%)$ & $479(55.0 \%)$ \\
Provincial and municipal funding & $90(43.9 \%)$ & $284(32.6 \%)$ \\
Others\# & $111(54.1 \%)$ & $458(52.6 \%)$ \\
None & $30(14.6 \%)$ & $211(24.2 \%)$ \\
\hline
\end{tabular}

*Two or more grant funds were acknowledged in some individual articles.

**Key Basic Research Foundation includes the National Basic Research Programme of China, the National High-tech R\&D Programme of China, and other national projects.

"This includes the Ministry of Education funding, institutional funding and international funding.

NSFC, funding from the National Natural Science Foundation of China.

Table 4. Top 10 journals that publish most articles by authors from Mainland Chinese hospitals

\begin{tabular}{|c|c|c|c|c|c|c|c|c|}
\hline \multirow[b]{2}{*}{ Rank } & \multicolumn{4}{|c|}{2012} & \multicolumn{4}{|c|}{2017} \\
\hline & Journal & $\begin{array}{l}\text { Impact } \\
\text { factor } \\
\text { in } 2017\end{array}$ & $\begin{array}{c}\text { Number of } \\
\text { Chinese } \\
\text { articles* }\end{array}$ & $\begin{array}{l}\text { Proportion } \\
(\%)\end{array}$ & Journal & $\begin{array}{l}\text { Impact } \\
\text { factor } \\
\text { in } 2017\end{array}$ & $\begin{array}{c}\text { Number of } \\
\text { Chinese } \\
\text { articles* }\end{array}$ & $\begin{array}{l}\text { Proportion } \\
\quad(\%)\end{array}$ \\
\hline 1 & Gastroenterology & 18.392 & $14(547)$ & 2.6 & Nature Communications & 12.124 & $73(4358)$ & 1.7 \\
\hline 2 & Hepatology & 13.246 & $13(537)$ & 2.4 & Hepatology & 13.246 & $67(511)$ & 13.1 \\
\hline 3 & Journal of Hepatology & 12.486 & $12(507)$ & 2.4 & Circulation & 19.309 & $44(793)$ & 5.5 \\
\hline 4 & Annals of Oncology & 11.855 & $12(601)$ & 2.0 & Gastroenterology & 18.392 & $37(576)$ & 12.2 \\
\hline 5 & Blood & 13.164 & $12(1682)$ & 0.7 & Cell Research & 15.606 & $29(159)$ & 18.2 \\
\hline 6 & European Heart Journal & 19.651 & $11(540)$ & 2.0 & Gut & 16.658 & $27(304)$ & 8.9 \\
\hline 7 & Journal of Pineal Research & 10.391 & $8(92)$ & 8.7 & ACS Nano & 13.942 & $25(1342)$ & 1.9 \\
\hline 8 & $\begin{array}{l}\text { Journal of the American College } \\
\text { of Cardiology }\end{array}$ & 19.896 & $8(934)$ & 0.9 & $\begin{array}{l}\text { New England Journal of } \\
\text { Medicine }\end{array}$ & 72.406 & $24(1486)$ & 1.6 \\
\hline 9 & Cell Research & 15.606 & $7(159)$ & 4.4 & $\begin{array}{l}\text { Journal of Allergy and } \\
\text { Clinical Immunology }\end{array}$ & 13.081 & $24(575)$ & 4.2 \\
\hline 10 & $\begin{array}{l}\text { American Journal of Respiratory } \\
\text { and Critical Care Medicine }\end{array}$ & 13.204 & $7(601)$ & 1.2 & Journal of Hepatology & 12.486 & $23(379)$ & 6.1 \\
\hline
\end{tabular}

*Total number of articles published in the year is listed in parenthesis.

The number of original articles significantly increased with a relatively decreased proportion. Also, the number of articles in basic research dramatically increased, with a stable proportion. Gastroenterology and hepatology was the top research field where authors from Mainland Chinese hospitals published articles in HIJs in both 2012 and 2017. Domestic and international cooperation was acknowledged in more articles published in 2017 than in 2012. The number (proportion) of articles published in NEJM, Lancet, JAMA, BMJ, Cell, Nature and Science all increased from 2012 to 2017. To the best of our knowledge, there are no earlier reports on the status and trend of research articles published in HIJs by authors from Mainland Chinese hospitals.

In 2017, the Institute of Scientific and Technical Information of China reported that the number of articles published in SCI journals by Chinese authors increased by almost two-fold from 2012 (approximately 170,000 articles) to 2016 (approximately 320,000 articles) ${ }^{4}$. This finding, along with our results, indicates that both the overall number of scientific articles and the number of 'high-quality' scientific articles published in HIJs by authors from Mainland Chinese hospitals have increased over the five-year period. However, only about $10 \%$ of these articles was published in the journals with JIF $>25$ in the present study, indicring that the quality of articles published by Mainland Chinese authors needs further improvement.

In the present study, the number of original articles increased by 3.5 -fold, with a decrease in proportion, and the number of articles in basic research increased by almost 4.4-fold with an unchanged proportion from 2012 to 2017. The number of articles in clinical research increased by 2.6-fold from 2012 to 2017 (89 vs 230), but the proportion was significantly reduced $(43.4 \%$ vs $26.4 \%$ ). These findings clearly demonstrate that the publication of original articles, especially in basic research, in HIJs has significantly increased from 2012 to 2017. Domestic and international cooperation and grant funding may have played an important role by promoting basic research. In the present study, although the overall number of cooperation-derived and funded articles increased 
Table 5. Articles published by authors from Mainland Chinese hospitals in the top 10 journals in terms of impact factor in 2017, the four popular general medical journals and Nature, Science and Cell in 2012 and 2017

\begin{tabular}{|c|c|c|c|c|c|c|}
\hline \multirow[b]{2}{*}{ Rank } & \multirow[b]{2}{*}{ Journal } & \multirow[b]{2}{*}{$\begin{array}{l}\text { Impact factor } \\
\text { in } 2017\end{array}$} & \multicolumn{2}{|c|}{2012} & \multicolumn{2}{|c|}{2017} \\
\hline & & & $\begin{array}{c}\text { Number of } \\
\text { Chinese articles* }\end{array}$ & Proportion $(\%)$ & $\begin{array}{c}\text { Number of } \\
\text { Chinese articles* }\end{array}$ & Proportion $(\%)$ \\
\hline 1 & CA: A Cancer Journal for Clinicians & 187.04 & $0(35)$ & 0.00 & $0(41)$ & 0.00 \\
\hline 2 & New England Journal of Medicine $e^{\#}$ & 72.406 & $0(1667)$ & 0.00 & $24(1486)$ & 1.60 \\
\hline 3 & Nature Reviews Drug Discovery & 57.000 & $1(156)$ & 0.60 & $1(135)$ & 0.70 \\
\hline 5 & Lancet $^{\#}$ & 47.831 & $4(1667)$ & 0.20 & $15(1488)$ & 1.00 \\
\hline 6 & Nature Reviews Molecular Cell Biology & 46.602 & $0(156)$ & 0.00 & $0(118)$ & 0.00 \\
\hline 7 & Journal of the American Medical Association ${ }^{\#}$ & 44.405 & $3(1014)$ & 0.30 & $17(1131)$ & 1.50 \\
\hline 8 & Nature Biotechnology & 41.667 & $0(255)$ & 0.00 & $0(242)$ & 0.00 \\
\hline 9 & Nature Reviews Genetics & 40.282 & $0(147)$ & 0.00 & $0(118)$ & 0.00 \\
\hline 10 & Nature & 40.137 & $1(2139)$ & 0.05 & $5(2193)$ & 0.20 \\
\hline 16 & Science & 37.205 & $0(1768)$ & 0.00 & $1(1687)$ & 0.06 \\
\hline 22 & Cell & 30.410 & $0(539)$ & 0.00 & $6(522)$ & 1.10 \\
\hline
\end{tabular}

*Total number of articles published in the year is listed in the parenthesis.

\#The four popular general medical journals.

significantly from 2012 to 2017 , the proportion remained similar, especially for original articles and those in basic research. Sun et al. ${ }^{10}$ reported that the ratio of funding articles in China was $77.8 \%$, much greater than USA, Germany, Britain, Japan, France, Italy, Canada, Spain and Australia. There was an average of 2.98 fund grants for each funded article published by authors from Mainland Chinese hospitals, which is higher than that in the countries listed above. In addition, it is believed that the dramatic increase in funding from NSFC, which primarily supports basic research, has also contributed to significant growth in the publication of original articles ${ }^{11,12}$. In the present study, NSFC funding consistently contributed to at least $55 \%$ of the articles. Funding from other sources also played an important role in the growth.

The present study shows that the research field of gastroenterology and hepatology ranked one in publishing articles by authors from Mainland Chinese hospitals in both 2012 and 2017. A major reason for this would be that the incidence of hepatitis, especially hepatitis B and $\mathrm{C}$, is high in China; so there has been extensive research on it ${ }^{13,14}$. Some journals in the field of materials science were included in the present study, mainly due to the fact that researchers from Mainland Chinese hospitals have long collaborated with those in materials science in biomedical research and publication.

We observed that the study designs of the original articles published in the four popular general medical journals are generally national surveys with significant medical importance ${ }^{15-21}$, prospective clinical studies with a large sample size ${ }^{22-24}$, and multicentre $\mathrm{RCTs}^{25-29}$; the authors were from different affiliations within China or even from other countries. Thus, domestic and international cooperation plays an important role for researchers from Chinese hospitals to publish in top journals.

\section{Conclusion}

Based on the above findings, we propose the following strategic recommendations. First, according to the findings of the original articles published in the four popular general medical journals, researchers may consider establishing and expanding domestic and international cooperation in order to carry out high-quality research. Second, NSFC funding consistently contributes to at least $55 \%$ of articles. So hospitals should give full support to researchers funded by NSFC to do basic research. In addition, funding from other sources was also acknowledged in over $50 \%$ of articles. So researchers should not ignore other funds. Third, original articles accounted for a small proportion of articles published in the four popular general medical journals, and majority were meta-analyses, letters, case reports, images, etc. Therefore, researchers may attempt to publish such types of articles in HIJs if there are insufficient resources to carry out large-scale, high-quality research.

Several limitations exist in the present study. First, only two databases, PubMed and Embase, were searched. However, these two are currently the most popular databases that cover all standard biomedical journals. Therefore, the present study would include almost all, if not all, research articles published by researchers from Mainland Chinese hospitals. Second, the top journals in some research fields, such as dentistry, dermatology, ophthalmology, orthopedics, otorhinolaryngology, etc. were not included as their JIF was not greater than 10. Therefore, the findings of the present study do not represent the research status in these fields.

In conclusion, the number of articles published in HIJs by authors from Mainland Chinese hospitals has increased rapidly from 2012 to 2017 . However, the number 
of articles published in journals with JIF $>25$ is still limited. The number of original articles in both basic and clinical research has also increased. Domestic and international cooperation and research funding are found to play an important role.

1. Journal impact factor; http://ipscience-help.thomsonreuters com/inCites2Live/indicatorsGroup/aboutHandbook/usingCitationIndicatorsWisely/jif.html (accessed on 22 May 2019).

2. Oh, H. C. and Lim, J. F., Is the journal impact factor a valid indicator of scientific value? Singapore Med. J., 2009, 50, 749 751

3. China's achievements shine 2017 scientific research 'world list', http://www.xinhuanet.com/mrdx/2017-12/20/c_136838875.htm (accessed on 22 May 2019)

4. ISI Journal Citation Reports, Institute for Scientific Information; http://isiknowledge.com (accessed on 22 May 2019).

5. Cheng, T., Research in orthopaedics from China has thrived over the last decade: a bibliometric analysis of publication activity. Orthop. Traumatol.: Sur. Res., 2012, 98, 253-258.

6. Wang, J. et al., Scientific publication in orthopedics journals from Chinese authors: a survey of 10-year survey of the literature. Eur. Spine J., 2015, 24, 1820-1825.

7. Liao, Q. J. et al., Perceptions of Chinese biomedical researchers towards academic misconduct: a comparison between 2015 and 2010. Sci. Eng. Ethics, 2018, 24, 629-645.

8. Statistical data with NSFC; http://www.nsfc.gov.cn/publish/ portal0/tab505/ (accessed on 22 May 2019)

9. Dan, F. and Yong, F., Role of SCI in the scientific research evaluation system of college-affiliated hospitals. Negative, 2016, 7, 56-59 (in Chinese)

10. Sun, J. W., Liu, D., Wang, X. W. and Hou, H. Y., Science funding and SCI papers output: a comparative analysis on 10 countries. Stud. Sci. Sci., 2013, 31, 36-42 (in Chinese).

11. Wang, X., Liu, D., Ding, K. and Wang, X., Science funding and research output: a study on 10 countries. Scientometrics, 2012, 91, 591-599.

12. Chen, Q. and Liu, H., Scientific research funding input and high level international papers output: the empirical analysis based on the SCI papers of 6 countries. Forum on Science Technology in China, 2018.

13. Zhang, Q. et al., Epidemiology of hepatitis B and hepatitis C infections and benefits of programs for hepatitis prevention in Northeastern China: a cross-sectional study. Clin. Infect. Dis., 2016, 62, 305-312.

14. Cui, Y. and Jia, J., Update on epidemiology of hepatitis B and C in China. J. Gastroenterol. Hepatol. (Suppl. 1), 2013, 28, 7-10.

15. Mok, T. S. et al., Osimertinib or platinum-pemetrexed in EGFR T790M-positive lung cancer. N. Engl. J. Med., 2017, 376, 629640.

16. Soria, J. C. et al., Osimertinib in untreated EGFR-mutated advanced non-small-cell lung cancer. N. Engl. J. Med., 2018, 378, $113-125$.
17. Robson, M. et al., Olaparib for metastatic breast cancer in patients with a germline BRCA mutation. N. Engl. J. Med., 2017, 377, 523-533.

18. Zhou, Y. et al., Tiotropium in early-stage chronic obstructive pulmonary disease. N. Engl. J. Med., 2017, 377, 923-935.

19. Xie, Y. L. et al., Evaluation of a rapid molecular drugsusceptibility test for tuberculosis. N. Engl. J. Med., 2017, 377, $1043-1054$

20. Zheng, M. X. et al., Trial of contralateral seventh cervical nerve transfer for spastic arm paralysis. N. Engl. J. Med., 2018, 378, 2234

21. Chan, K. C. A. et al., Analysis of plasma Epstein-Barr virus DNA to screen for nasopharyngeal cancer. N. Engl. J. Med., 2017, 377, 513-522.

22. Zhang, L. et al., Prevalence of chronic kidney disease in China: a cross-sectional survey. Lancet, 2012, 379, 815-822.

23. Lu, J. et al., Prevalence, awareness, treatment, and control of hypertension in China: data from 1.7 million adults in a population-based screening study (China PEACE Million Persons Project). Lancet, 2017, 390, 2549-2558.

24. Su, M. et al., Availability, cost, and prescription patterns of antihypertensive medications in primary health care in China: a nationwide cross-sectional survey. Lancet, 2017, 390, 2559-2568.

25. Tan, J. et al., Induction therapy with autologous mesenchymal stem cells in living-related kidney transplants: a randomized controlled trial. JAMA-J. Am. Med. Assoc., 2012, 307, 11691177.

26. Liu, Z. et al., Effect of electroacupuncture on urinary leakage among women with stress urinary incontinence: a randomized clinical trial. JAMA - J. Am. Med. Assoc., 2017, 317, 2493-2501.

27. Lv, J. et al., Effect of oral methylprednisolone on clinical outcomes in patients with $\operatorname{IgA}$ nephropathy: the testing randomized clinical trial. JAMA - J. Am. Med. Assoc., 2017, 318, $432-442$.

28. Wang, H. et al., Effect of levothyroxine on miscarriage among women with normal thyroid function and thyroid autoimmunity undergoing in vitro fertilization and embryo transfer: a randomized clinical trial. JAMA - J. Am. Med. Assoc., 2017, 318, 2190-2198.

29. Wu, X. K. et al., Effect of acupuncture and clomiphene in Chinese women with polycystic ovary syndrome: a randomized clinical trial. JAMA - J. Am. Med. Assoc., 2017, 317, 2502-2514.

ACKNOWLEDGEMENTS. We thank our colleagues in Medjaden Bioscience Limited, Wuhan, China, for downloading the full texts of articles and collecting data. The results were orally presented at the Second International Summit on Medical Research and Publication, and The First International Forum on Frontiers in Medicine during 1516 September 2018 at Wuhan, China (http://www.mrpcenter.com/ ismrp/).

Received 22 May 2019; revised accepted 9 August 2019

doi: $10.18520 / \mathrm{cs} / \mathrm{v} 117 / \mathrm{i} 11 / 1793-1799$ 